\title{
Radiation induces proinflammatory dysbiosis: transmission of inflammatory susceptibility by host cytokine induction
}

\author{
Shiran Gerassy-Vainberg, ${ }^{1,2}$ Alexandra Blatt, ${ }^{1}$ Yael Danin-Poleg, ${ }^{2}$ Katya Gershovich, ${ }^{1}$ \\ Edmond Sabo, ${ }^{3}$ Alex Nevelsky, ${ }_{6}^{4}$ Shahar Daniel, ${ }^{4}$ Aviva Dahan, ${ }^{1}$ Oren Ziv, ${ }^{5}$ \\ Rishu Dheer, ${ }^{6}$ Maria T Abreu, ${ }^{6}$ Omry Koren, ${ }^{5}$ Yechezkel Kashi, ${ }^{2}$ Yehuda Chowers ${ }^{1}$
}

Additional material is published online only. To view please visit the journal online (http://dx.doi.org/10.1136/ gutjnl-2017-313789)

For numbered affiliations see end of article.

Correspondence to Dr Yehuda Chowers, Department of Gastroenterology, Rambam Health Care Campus, Haifa 3109601, Israel: ychowers@gmail.com

Received 23 January 2017 Revised 20 March 2017 Accepted 3 April 2017 Published Online First 24 April 2017

\section{SLinked}

- http://dx.doi.org/10.1136/ gutjnl-2017-314279

CrossMark

To cite: Gerassy-Vainberg $\mathrm{S}$, Blatt A, Danin-Poleg Y, et al. Gut 2017;67:97-107.

\section{ABSTRACT}

Objective Radiation proctitis (RP) is a complication of pelvic radiotherapy which affects both the host and microbiota. Herein we assessed the radiation effect on microbiota and its relationship to tissue damage using a rectal radiation mouse model.

Design We evaluated luminal and mucosa-associated dysbiosis in irradiated and control mice at two postradiation time points and correlated it with clinical and immunological parameters. Epithelial cytokine response was evaluated using bacterial-epithelial co-cultures. Subsequently, germ-free (GF) mice were colonised with postradiation microbiota and controls and exposed to radiation, or dextran sulfate-sodium (DSS). Interleukin (IL)-1 $\beta$ correlated with tissue damage and was induced by dysbiosis. Therefore, we tested its direct role in radiation-induced damage by IL-1 receptor antagonist administration to irradiated mice.

Results A postradiation shift in microbiota was observed. A unique microbial signature correlated with histopathology. Increased colonic tumor necrosis factor (TNF) $\alpha$, IL- $1 \beta$ and IL- 6 expression was observed at two different time points. Adherent microbiota from RP differed from those in uninvolved segments and was associated with tissue damage. Using bacterial-epithelial co-cultures, postradiation microbiota enhanced IL-1 $\beta$ and TNF $\alpha$ expression compared with naïve microbiota. GF mice colonisation by irradiated microbiota versus controls predisposed mice to both radiation injury and DSSinduced colitis. IL-1 receptor antagonist administration ameliorated intestinal radiation injury.

Conclusions The results demonstrate that rectal radiation induces dysbiosis, which transmits radiation and inflammatory susceptibility and provide evidence that microbial-induced radiation tissue damage is at least in part mediated by IL-1 $\beta$. Environmental factors may affect the host via modifications of the microbiome and potentially allow for novel interventional approaches via its manipulation.

\section{INTRODUCTION}

Radiation therapy may lead to acute and chronic toxicities that limit its use. The intestine is particularly radiosensitive, due to the rapid gut epithelial turnover. $^{1-3}$

Radiation-induced tissue damage in humans was shown to be associated with elevated levels of

\section{Significance of this study}

What is already known on this subject?

- Therapeutic radiation may cause severe intestinal damage. Radiation exposure induces apoptosis of epithelial cells, followed by mucosal inflammation, and is associated with ulceration, fibrosis and a disorganised process of tissue vascularisation, resulting in diarrhoea, bleeding, pain and impaired quality of life.

- Interleukin (IL)-1 $\beta$ was previously shown to best correlate with radiation-induced intestinal damage in both humans and mice, yet the detailed interaction between IL-1 $\beta$ and radiation injury is poorly defined.

- Changes in gut microbiota composition following radiotherapy were reported in several radiation-associated conditions. The major changes observed were reduced diversity of the Firmicutes and Bacteroidetes and increase in Proteobacteria. However, no information is available regarding the effect of radiation-induced dysbiosis on specific pathogenic mechanisms of postradiation enteritis.

\section{What are the new findings?}

- Radiation treatment induced localised microbial dysbiosis. Mucosa associated dysbiosis was correlated with local tissue damage but did not occur in intact tissues.

- Radiation-induced dysbiotic microbiota transmitted inflammatory susceptibility and rendered germ-free recipient mice more susceptible to radiation damage and dextran sulfate sodium-induced colitis.

- Radiation-induced microbiota instigated increased secretion of IL-1 $\beta$ both in bacterial-epithelial co-cultures and in vivo in the colonised germ-free mice. Blocking IL-1 showed that IL-1 was a major driver of tissue damage in radiation proctitis.

How might it impact on clinical practice in the foreseeable future?

- The findings suggest that microbiota manipulation or direct inhibition of IL-1 should be considered as potential clinical interventions to ameliorate or prevent radiation-induced tissue damage and thereby provide therapeutic benefit. 
interleukin 2 (IL-2), IL-6, IL-8 and particularly IL-1 $\beta .{ }^{4}$ We have previously shown that IL- $1 \beta$ was also most closely correlated with tissue injury in a mouse radiation proctitis (RP) model. ${ }^{5}$

Previous attempts to minimise radiation injury included use of free radical scavengers, and improved radiation techniques. ${ }^{6-8}$ However, currently, the main approach to reduce injury is reduction of the delivered radiation dose. This practice may lead to decreased treatment efficacy. ${ }^{9-11}$ Better understanding of radiation-induced mucosal pathogenesis may reveal additional relevant pathogenic factors, potentially leading to more detailed mechanistic understanding.

Radiation treatment may affect the patient's tissue and the entire intestinal milieu. In support of such a possibility, a shift in gut microbiota composition following radiotherapy was previously shown in mouse models of segmental small bowel radiation $^{12}$ and graft versus host disease (GVHD), ${ }^{13} 14$ and in human pathologies consisting of acute postradiotherapy diarrhoea, ${ }^{15} \mathrm{GVHD}^{14}$ and postradiation patients with gynaecological cancer. ${ }^{16} 17$ However, hitherto, no causal effect was attributed to radiation-induced dysbiosis.

Herein, we used a mouse model of localised directed rectal radiation to investigate the dynamics of the intestinal microbiota, its interplay with the host immune system and its role in radiation-induced tissue damage.

\section{MATERIALS AND METHODS Irradiated mouse model}

Female C57BL/6J mice aged 6-8 weeks (Harlan Laboratories, Israel) were maintained under specific pathogen-free (SPF) conditions with free access to drinking water and food. Mice were treated with localised internal rectal radiation using MultiSource HDR afterloader (Eckert \& Ziegler BEBIG GmbH, Germany) as previously described. ${ }^{5}$

Irradiation procedures and IL-1 receptor antagonist (anakinra) administration are detailed in the online supplementary material.

Animal studies were approved by the animal care committee of the Technion-Israel Institute of Technology, Haifa, Israel (IL-073-06-2011 and IL-088-08-2015).

\section{Sample collection}

Stool was sampled at several time points: 1 week before radiation and 2 and 6 weeks postradiation. Samples from each mouse were either stored at $-80^{\circ} \mathrm{C}$ for microbiota composition analysis or processed for further experimentation. For this purpose, samples were homogenised with phosphate - buffered saline (PBS) (Biological Industries) (10\% weight/volume), mixed by vortex and centrifuged for $5 \mathrm{~min}$ at $800 \mathrm{rpm}$ and bacterial cells were pelleted. Pellets were washed in PBS, resuspended in an equal volume of $50 \%$ glycerol with brain heart medium (Difco) and stored at $-80^{\circ} \mathrm{C}$ for co-culture experiments.

Colonic segments were immediately frozen in liquid nitrogen and stored at $-80^{\circ} \mathrm{C}$. Tissues were subsequently analysed for adherent microbiota composition and local cytokine expression. A parallel colon segment was fixed in $4 \%$ formaldehyde for histopathological analysis.

\section{Cytokine expression}

RNA was extracted from colon samples using RNeasy Mini Kit (QIAGEN). Complementary DNA was synthesised using an oligo $d(T)$ primer from the Verso cDNA kit (Thermo Scientific). Real-time quantitative PCR was performed using a StepOnePlus Real-Time PCR System (Life Technologies). Relative cytokine expression was calculated following normalisation to phosphoglycerate kinase expression. Cytokine mRNA expression in co-culture experiments was normalised to glyceraldehyde-3 phosphate dehydrogenase expression. The PCR primer sets are described in online supplementary table S1. Primers were purchased from Sigma Aldrich.

\section{Colonic IL-1ß quantification by ELISA}

Total protein was extracted from colonic tissue using an extraction kit (Merck Millipore) according to the manufacturer's instructions.

IL-1 $\beta$ was quantified using an IL-1 $\beta$ Mouse ELISA Kit (Abcam) according to the manufacturer's instructions.

\section{Histopathology}

Colon tissue samples were fixed in 4\% formaldehyde, processed and stained with H\&E. Samples were assessed blindly by an experienced GI pathologist. Radiation histopathology results were expressed by the semiquantitative radiation injury score (RIS) comprised of seven histopathological categories (maximum score is 18) as previously described ${ }^{18} 19$ and an additional parameter of inflammation assessing inflammatory cell infiltration.

Dextran sulfate sodium (DSS)-induced colitis evaluation was performed using a combined inflammatory histological score (maximum score is 11 ) as previously described. ${ }^{20}$

\section{Microbiota composition analysis}

Next-generation amplicon sequencing

Faecal DNA samples of conventionally raised SPF C57Bl/6 mice were sequenced at the Research and Testing Laboratory (Lubbock, Texas, USA) using Roche 454 Pyrosequencing. Sequence reads are available under NCBI BioProject PRJNA283782 (http://www.ncbi.nlm.nih.gov/bioproject/283782).

DNA of mucosa-associated microbiota in colonic segments was sequenced at the DNA Services Facility-University of Illinois at Chicago using Illumina Miseq platform. Sequence reads are available under NCBI BioProject PRJNA353641 (http://www.ncbi.nlm.nih.gov/bioproject/353641).

Faecal DNA samples of Swiss Webster germ-free (GF) mice postinoculation and radiation were also sequenced at the DNA Services Facility-University of Illinois at Chicago using Illumina Miseq platform. Sequence reads are available under NCBI BioProject PRJNA342751 (https://www.ncbi.nlm.nih.gov/ bioproject/342751).

Data were processed using QIIME V. 1.8.0 (Quantitative Insight Into Microbial Ecology) pipeline. ${ }^{21}$ For details, see online supplementary material.

\section{Epithelial cell-faecal microbiota co-culture}

Human HT29 colonic epithelial cells were obtained from American Type Culture Collection (Manassas, Virginia, USA) and maintained as previously described. ${ }^{22}$ Cells (passage 34) were seeded at a density of $5 \times 10^{4}$ cells $/ \mathrm{cm}^{2}$ onto 24 -well plates in McCoy medium (Biology industries) supplemented with 10\% heat inactivated foetal calf serum (Biological Industries) and $2 \mathrm{mM}$ glutamine and grown at $37^{\circ} \mathrm{C}$ in $5 \% \mathrm{CO}_{2}$ and $95 \%$ air for 24 hours. For co-culture, the medium was changed and bacterial cell suspension obtained from irradiated and sham control mice was adjusted to reach an optical density of $0.05\left(\mathrm{~A}_{\lambda 600}\right)$. A $20 \mu \mathrm{L}$ working suspension was added to each well of a 24-well plate. The total culture volume was $1 \mathrm{~mL}$. Co-cultures were incubated at $37^{\circ} \mathrm{C}$ for $6-7$ hours under microaerophilic conditions. Cytokine expression by colonic epithelial cells was assessed as described above. 


\section{GF mouse model experiments}

GF mouse model of RP

Mouse inoculation was performed by suspending one stool pellet from radiation-treated and naive mice in $1 \mathrm{~mL}$ of sterile PBS. A volume of $200 \mu \mathrm{L}$ faecal suspension was introduced to recipient GF Swiss Webster mice by oral gavage. Each transplanted mouse received an inoculum from a different donor mouse. Transplanted mice were rectally irradiated 14 days postinoculation as described above. Mice were sacrificed 2 weeks postradiation. Colonic segments were tested for histology and cytokine quantification as detailed above.

\section{GF mouse model of DSS colitis}

Faecal samples from irradiated and naïve mice were suspended in $25 \%$ glycerol diluted in PBS (10\% weight/volume) as described above. GF C57Bl/6 mice were orally gavaged with $200 \mu \mathrm{L}$ faecal suspension. Mice were treated 2 weeks postinoculation with $2.5 \%$ DSS in drinking water for 6 days. Mice were monitored daily to determine Disease Activity Index (DAI) as previously described. ${ }^{23}$ Colonic segments were harvested at the experimental end point and tested for histological evaluation using combined inflammatory histological scores.

\section{Data analysis}

Faecal microbiota composition analyses were performed using QIIME (V.1.8.0) pipeline. $^{21} \beta$ diversity was determined by computing unweighted and weighted UniFrac ${ }^{24}$ distance. The resulting matrices were visualised by principal coordinates analysis (PCoA) plots. Distributions of distances were compared with two-tailed, two-sample t-tests.

Mucosa-associated microbiota was also plotted in weighted UniFrac-based PCoA. PCo score comparison based on the Kruskal-Wallis test was used to test for differences in community composition among the different groups. Heatmap based on pyrosequencing results at the genus level of faecal microbiota at different time points postradiation was generated using the 'heatmap' module of the statistical software environment R. Data were normalised to the average frequency in control mice in each clinical stage and scaled internally on a row basis to a mean of 0 and a SD of 1 . The hierarchical clustering was based on Euclidian distance and word's agglomeration method (boot $n=1000$ ). Linear discriminant analysis (LDA) effect size (LEfSe) was used to identify differences in relative abundance at different taxonomic levels. ${ }^{25}$

Comparisons of cytokine expression and histological scores were performed by two-tailed (Wilcoxon) Mann-Whitney U test. Correlations were calculated using Pearson's correlation coefficient.

\section{RESULTS \\ Locally delivered rectal radiation induces inflammation, colonic tissue injury and inflammatory cytokine expression mimicking human RP}

In the current study, we took advantage of our previously developed mouse RP model using localised rectal radiation. ${ }^{5}$ Radiation-induced colonic damage was evaluated using histology (RIS) and inflammation score) and colonic mRNA cytokine expression. The mRNA (figure 1A) of IL-1 $(2$ and 6 weeks: $p<0.05$, Mann-Whitney $U$ test), IL-6 (2 weeks: $\mathrm{p}<0.05$; 6 weeks: $\mathrm{p}<0.01$, Mann-Whitney $\mathrm{U}$ test) and TNF $\alpha$ ( 2 and 6 weeks: $p<0.01$, Mann-Whitney $U$ test) was significantly higher in irradiated mice compared with control mice, both at 2 and 6 -week postradiation time points.
Histopathological assessment demonstrated that cytokine expression paralleled tissue damage. Inflammation and RIS were significantly higher in irradiated mice 2 and 6 weeks posttreatment compared with controls $(\mathrm{p}<0.0001$, Mann-Whitney U test, figure $1 \mathrm{~B})$.

Although both the 2 and 6 -week postradiation time points were characterised by inflammation, analysis of the overall inflammatory parameters, including colonic cytokine expression and histological scores by principal component analysis (figure 1C), demonstrated a difference between these time points, corresponding to acute and chronic phases of the disease. The most prominent differences between time points were in the inflammatory score and expression of IL-1 $\beta$ and IL-6 (figure 1C). As presented in online supplementary table S2, both IL-1 $\beta$ and TNF $\alpha$ were correlated with the RIS $(R=0.6$, $\mathrm{p}=0.001$ ); however, a stronger correlation with the histological inflammatory score was noted with IL- $1 \beta(R=0.4, p<0.05)$, suggesting its importance in response to radiation.

\section{$\mathrm{RP}$ is associated with dysbiosis characterised by a unique microbial signature at each time point}

To evaluate the impact of radiation treatment on the gut bacterial population, faecal samples (experiment group $n=23$, control group $\mathrm{n}=22$ ) of pretreatment, 2 and 6 weeks postradiation were analysed by 454 pyrosequencing. Bacterial communities of irradiated and control mice were compared using unweighted and weighted UniFrac-based PCoA (figure 2A). A significant shift in postradiation gut microbial composition was noted $(q<0.0001)$, suggesting that targeted local radiation dramatically altered gut microbial composition.

Analysis of microbial composition at the specific time points revealed a unique microbial stage-related signature (figure $2 \mathrm{~B}$ ). The most significant shift in microbial composition was observed 6 weeks postradiation. This may indicate a trend of gradual microbial response to radiation exposure and the consequent alternations in the intestinal environment. These results also illustrate the parallel postradiation course of microbial dynamics and colonic inflammation.

LEfSe was used to identify bacterial taxa associated with radiation-induced tissue damage (figure 3). As shown, three out of the six dominant bacterial phyla showed a significant change in abundance between irradiated mice compared with controls. These included the Proteobacteria phylum to which Suterella spp. belong (LDA score $=3.1, \mathrm{p}<0.0001$ ) and Verucomicrobia phylum including the Akkermansia spp. (LDA score=3.6, $\mathrm{p}<0.01)$. Radiation significantly reduced the prevalence of Firmicutes (LDA score $=4.1, \mathrm{p}<0.01)$. Six genera showed increased abundance in mice that developed RP compared with control mice (LDA score $>2.5, \mathrm{p}<0.05$ ) including Akkermansia, Bacteroides, Parabacteroides, Sutterella, Turicibacter and an unclassified genus belonging to the RF32 order.

The adherent bacterial population was next tested in different colonic segments. Bacterial communities of inflamed (rectum) and intact (proximal colon) tissues were assessed by Illumina sequencing 6 weeks postradiation. Uninflammed segments were taken $2 \mathrm{~cm}$ above the radiated area (inflammation was confirmed by histology and cytokine expression). Histological scores for distal inflamed and proximal uninflamed colonic segments are shown in online supplementary table S3.

Comparison of the microbial composition between irradiated and naïve mice based on weighted Unifrac PCoA demonstrated a significant change between irradiated distal colon and the distal colon of naïve mice. No significant difference was observed in the proximal colonic segment between the two 

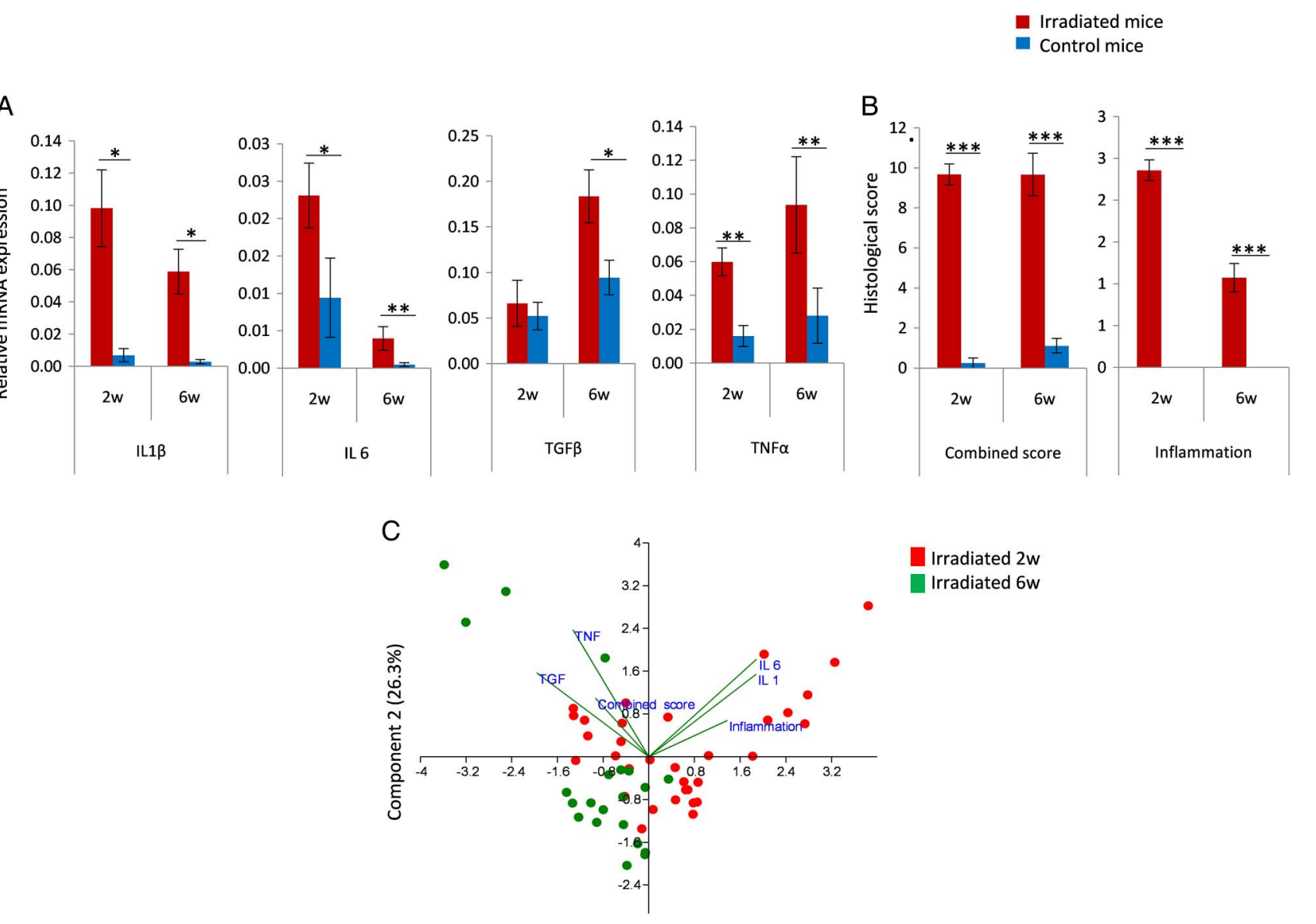

Component $1(35.2 \%)$

Figure 1 Evaluation of postradiation colonic damage in C57BL/6J mice. (A) Colonic cytokine expression (IL-1 $\beta$, IL-6, TGF $\beta$ and TNF $\alpha$ ) was assessed by quantitative PCR using mRNA extracted from colon biopsy samples. (B) Postradiation tissue damage was assessed by the semiquantitative radiation injury score and scoring of inflammation. Experimental and naïve groups are represented in red and blue columns respectively, as mean \pm SEM (experiment group: 2 weeks $n=33,6$ weeks $n=21$; naïve group: 2 weeks $n=8,6$ weeks $n=9$, data were pooled from three independent experiments). ${ }^{*} p<0.05 ;{ }^{* *} p<0.01 ;{ }^{* *} p<0.001$, Mann-Whitney $U$ test. (C) Principal component analysis (PCA) of the inflammatory state demonstrates distinct clustering at 2 and 6 weeks postradiation. Overall inflammatory parameters (cytokine expression and histological scores) were compared and clustered based on correlation index measurement using two-dimensional PCA. The difference in the inflammatory state of irradiated mice at 2 weeks (red) and 6 weeks (green) postradiation was significant (analysis of similarities (ANOSIM) comparison: R=0.4, $\mathrm{q}<0.0001$ ). IL, interleukin.

mouse groups (irradiated vs naïve distal colons: $\mathrm{p}<0.05$; irradiated vs naive proximal colons $\mathrm{p}=\mathrm{NS}, \mathrm{PCo} 3$ score comparison in weighted Unifrac indices, Kruskal-Wallis, figure 4). This observation suggested that the microbial changes were associated with local tissue damage. Notably, following radiation the microbiota of the irradiated distal colon became more similar to that of the proximal unirradiated segment, leading to loss of the distinctive microbial composition noted in the two colonic sites of naïve mice.

Collectively, the results demonstrate that gut microbiota composition is associated with the changing inflammatory intestinal milieu and radiation-induced tissue damage. We next addressed the cause and effect relationship between these two components.

\section{Radiation-altered microbiota induces increased}

\section{inflammatory responses in intestinal epithelial cultures}

An in vitro epithelial cell co-culture model was used to explore the potential effects of radiation-induced microbial dysbiosis on the epithelial inflammatory response. HT29 epithelial cells were co-cultured with faecal bacterial suspensions from irradiated and naïve mice obtained 6 weeks postradiation. Epithelial cytokine expression following co-culture with faecal suspensions from control naïve mice and radiated mice were compared (figure 5). Radiation-induced faecal microbiota induced TNF $\alpha$ (2.4-fold, $\mathrm{p}<0.05$, Mann-Whitney U test) and IL-1 $\beta$ secretion (1.7-fold, $\mathrm{p}<0.01$, Mann-Whitney $U$ test) from the epithelial cells compared with naïve microbiota. Notably, co-incubation of the faecal bacteria with HT29 cells was performed under microaerophilic conditions. This was done as a compromise between the anaerobic environment required by the bacteria and aerobic conditions needed for optimal growth of epithelial cells. Incubation time was limited to 6-7 hours.

These results support the contention that compositional changes in the microbiota resulting from radiation treatment impact epithelial cells in a manner that may be associated with the pathogenesis of radiation-induced damage.

\section{Radiation-induced dysbiosis transmits susceptibility to RP}

To further assess the in vivo impact of radiation-induced microbiota on colonic tissue injury, we inoculated GF mice with faecal microbiota obtained from irradiated and naïve mice and exposed them to radiation. Mice inoculated with radiation-induced microbiota exhibited more severe pathological features of radiation damage compared with mice inoculated 

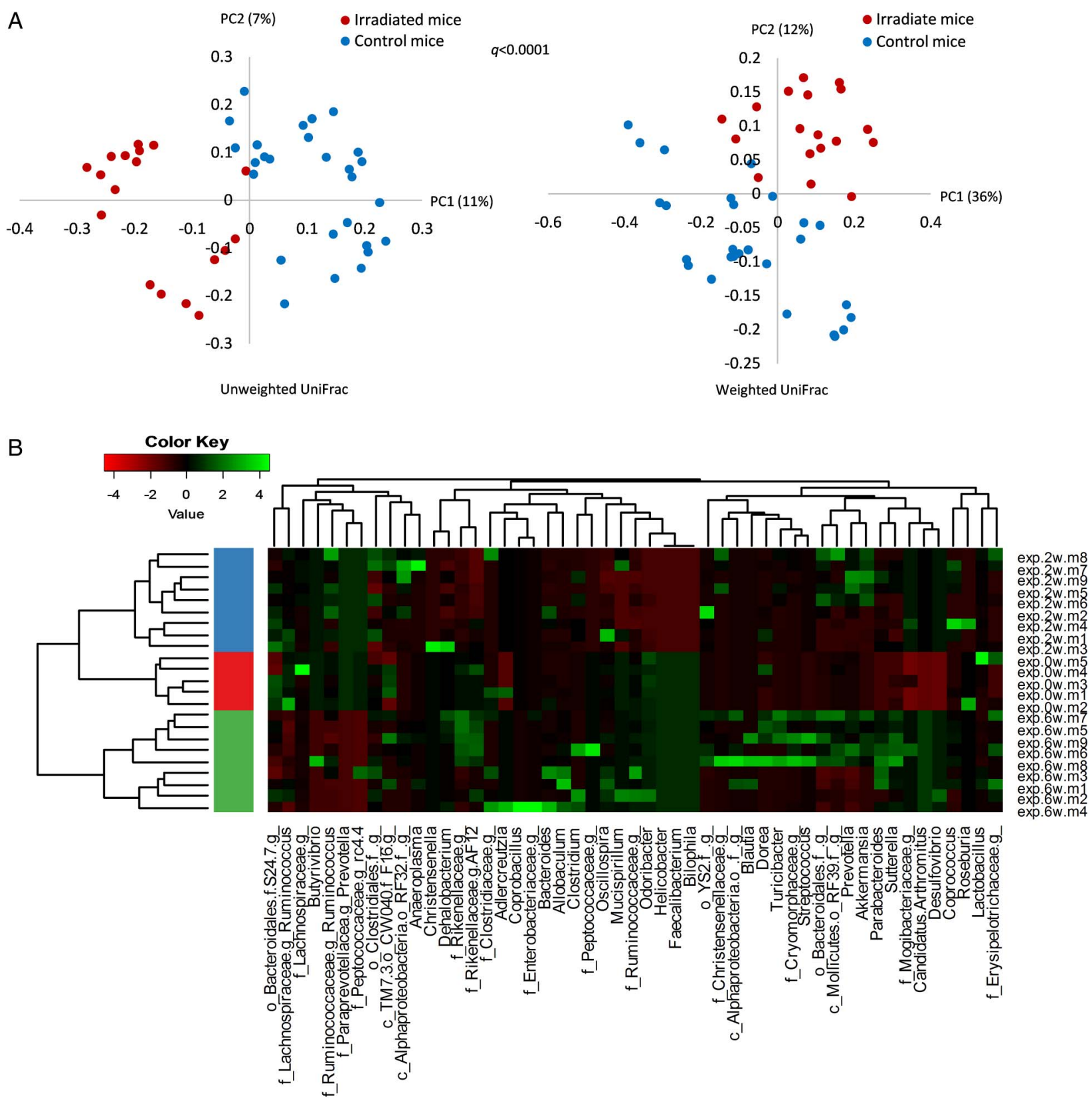

Figure 2 Faecal microbiota composition alternation following radiation treatment at various time points. Bacterial composition was assessed by 16S rRNA sequencing of faecal samples using 454 pyrosequensing. (A) Distances between bacterial communities were clustered using principal coordinates analysis based on unweighted and weighted UniFrac measure. Irradiated mice ( 2 weeks: $n=9 ; 6$ weeks: $n=9$ ): red dots; control mice ( 0 weeks: $n=9 ; 2$ weeks: $n=9 ; 6$ weeks: $n=9$ ): blue dots. Data were pooled from two independent experiments, conducted nearly a year apart. (B) Microbial dynamics at different time points presented by heatmap. The major bacterial genera frequency (vertical line) at different time points of radiation proctitis was normalised to the average frequency in control mice in each time point. Horizontal lines represent faecal samples obtained from each mouse at each time point, ordered by cluster analysis. The fold-change in bacterial abundance of each genus is expressed in SD units scaled internally on a row basis and represented by a colour key. Hierarchical clustering was based on Euclidian distance measure and word's agglomeration method (boot $n=1000$ ). The left colour bar in the heatmap indicates microbial community classes as defined by hierarchical clustering. Samples were collected at three time points: before irradiation, 2 and 6 weeks postirradiation. Samples were clustered and marked red, blue and green correspondingly.

with naïve microbiota as assessed by the RIS $(p<0.005$, onetailed t-test; figure 6A). Notably, no significant difference was noted in the inflammation subscore, suggesting that cellular infiltration did not play a significant role at this stage of the process.

Cytokine expression analysis also demonstrated a difference between the postradiation treated groups ( $\mathrm{PCo} 3$ scores comparison, $\mathrm{p}<0.05$; one-tailed $\mathrm{t}$-test; figure $6 \mathrm{~B}$ ). IL- $1 \beta$ expression was the major contributor to the difference between groups based on factor loading calculation (IL-1 $\beta$ factor loading on PC3: 0.97). Further analysis demonstrated a significant increase in IL-1 $\beta$ protein level $(\mathrm{p}<0.05$, one-tailed $\mathrm{t}$-test, figure $6 \mathrm{E})$. Notably, although increased TNF $\alpha$ expression was observed following radiation in SPF mice compared with controls and in epithelial cells/ bacterial co-cultures with postradiation microbiota in vitro, no 
Figure 3 Bacterial markers associated with radiation proctitis (RP), at 2 and 6 weeks postradiation. Differentially abundant operational taxonomic units (OTUs) between irradiated (red) and control mice (blue) associated with RP were identified using linear discriminant analysis (LDA effect size) and presented by a histogram of LDA scores. Groups were defined according to the microbiota origin. Irradiated versus control mouse subgroups were defined according to sampling time: 2 and 6 weeks postradiation. Only OTUs that significantly differed in both group and subgroup comparisons were detected.
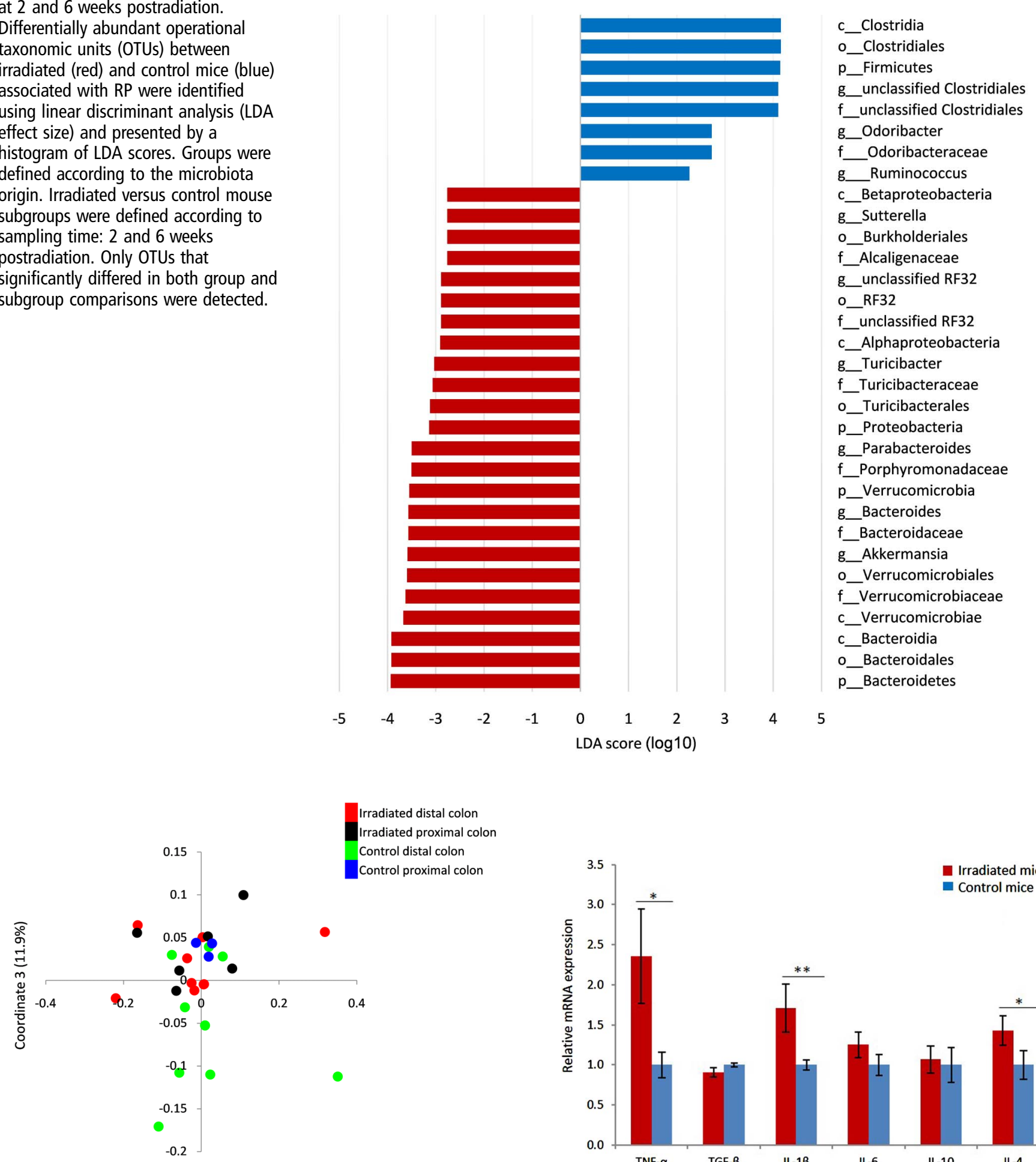

Coordinate 1 (41.9\%)

Figure 4 Mucosa-associated microbiota dynamics following localised radiotherapy. Bacterial composition was assessed by $16 \mathrm{~s}$ rRNA using Illumina Miseq platform. Principle coordinates analysis of microbiota diversity was performed based on weighted Unifrac measure. Each point represents the bacterial profile of a single mouse at a specific colonic segment. The control group consisted of healthy mice tested in parallel colonic segments. The microbiota composition of distal $(n=9$; red dots) and proximal colon ( $n=6$; black dots) of irradiated mice; and distal ( $n=9$; green dots) and proximal ( $n=3$; blue dots) colon of control mice were tested.

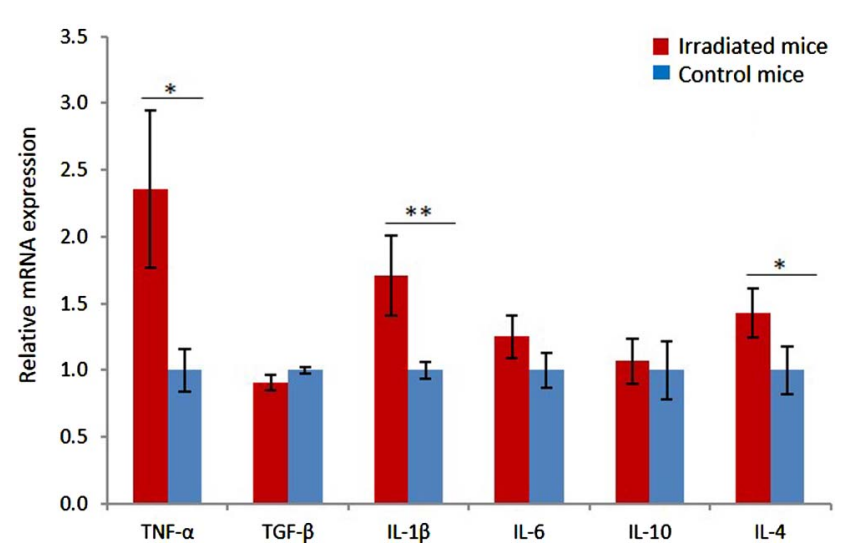

Figure 5 Differential cytokine expression from HT-29 epithelial cells induced by co-culture with irradiated microbiota. Faecal bacterial suspensions obtained from irradiated mice 6 weeks postradiation (red) or from age matched controls (blue) were co-cultured with HT-29 epithelial cells for 6-7 hours. Cytokine expression was assessed using mRNA quantitative PCR. Each bacterial suspension was prepared as a pool from at least seven mice tested at two independent radiation trials. Co-incubation experiments from each trial were repeated five to six times. Data were normalised to the average mRNA cytokine expression of epithelial cells co-cultured with bacteria from naïve mice. ${ }^{*} \mathrm{p}<0.05 ;{ }^{* *} \mathrm{p}<0.01$, Mann-Whitney U test. 
A

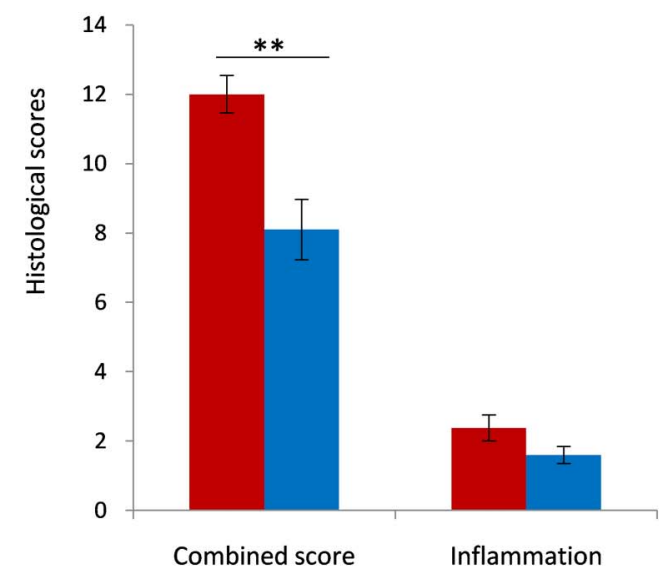

C

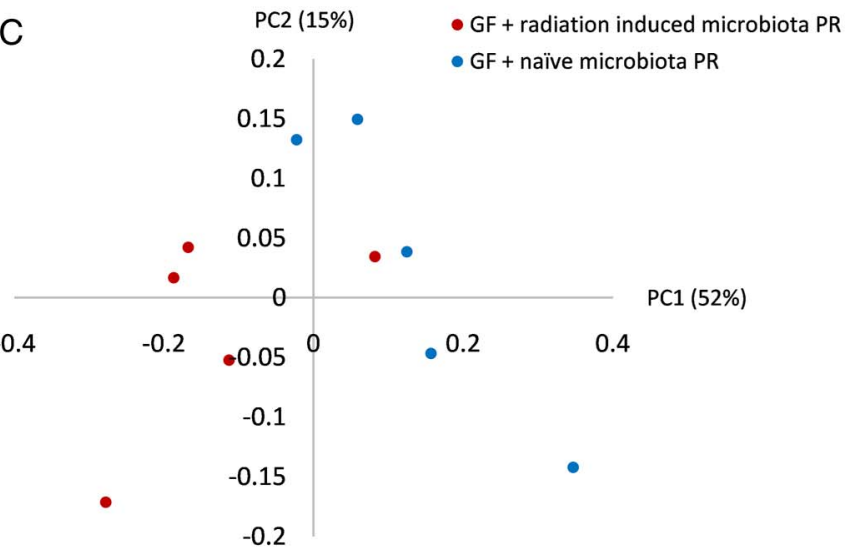

$\mathrm{E}$

- GF + radiation-induced microbiota

n GF + naïve microbiota

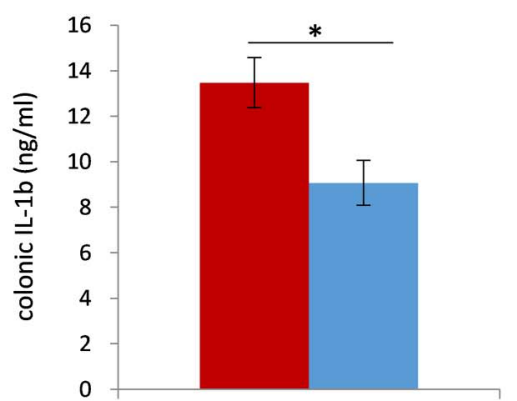

GF + radiation induced microbiota $\mathrm{PR}$ GF + naïve microbiota $P R$
B

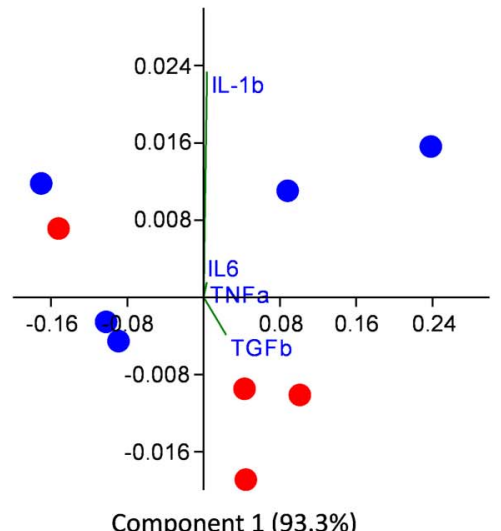

Component 1 (93.3\%)

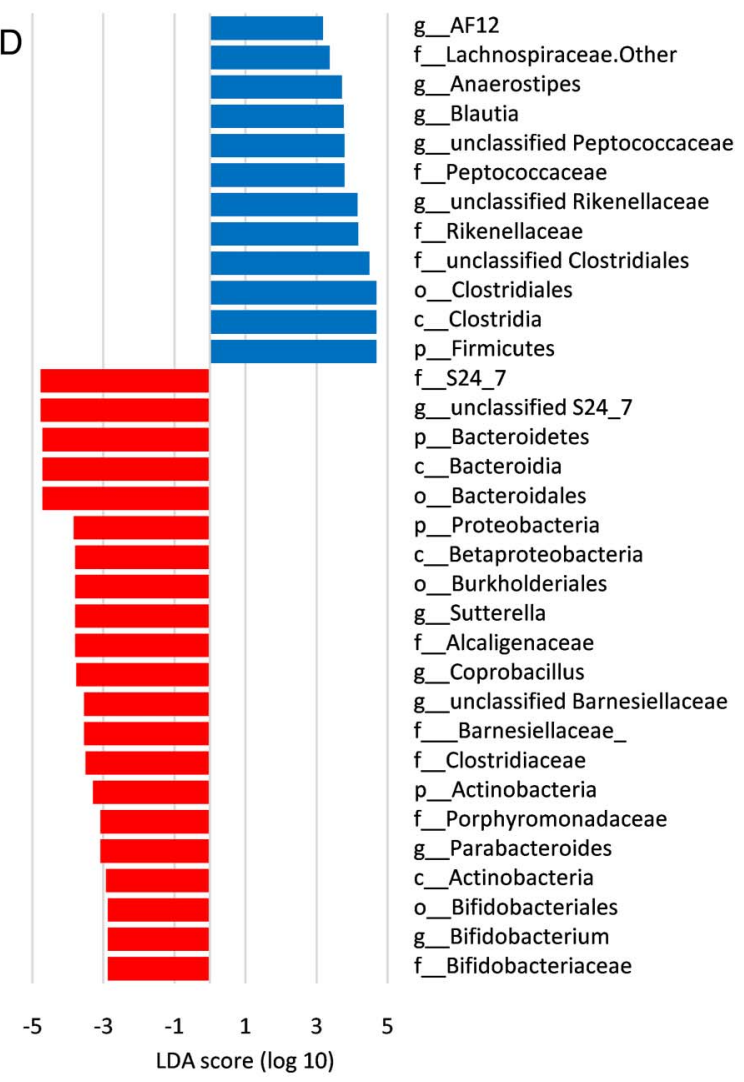

D

Figure 6 Radiation-induced microbiota worsens radiation-induced colonic tissue injury in vivo. (A) Postradiation histological assessment of germ-free (GF) mice inoculated with faecal microbiota from irradiated (red columns) and naïve mice (blue columns). Histology was assessed by the semiquantitative radiation injury score and inflammation scoring. Histological scores were evaluated 2 weeks postradiation and are represented as mean \pm SEM (GF+radiation-induced microbiota: $n=5 ; G F+$ naïve group: $n=4)$ ) * *indicates $p \leq 0.01$, one-tailed t-test (B). Colonic cytokine expression profile is plotted against coordinate $1(93 \%)$ and coordinate $3(1 \%)$. Differences were observed between GF mice inoculated with irradiated and naïve microbiota (PC03 score comparison, $p<0.05$; one-tailed $t$-test). (C) Faecal microbiota composition from GF mice inoculated with microbiota from irradiated and naive donors after radiation treatment of both groups was assessed by $16 \mathrm{~S}$ rRNA sequencing using the Illumina Miseq platform and compared by PCo score comparison: $\mathrm{p}<0.05$, PCo1 score comparison in weighted UniFrac index, Mann-Whitney U test. (D) Differentially abundant operational taxonomic units associated with RP were identified using linear discriminant analysis (LDA effect size) in GF mice inoculated with microbiota from irradiated and naïve donors postinoculation and radiation. Groups were defined according to the microbiota origin; subgroups were defined according to sampling time: 2 weeks postinoculation, before radiation and 2 weeks postradiation. (E) Colonic IL-1 $1 \beta$ protein level in GF mice inoculated with microbiota from irradiated (red) and naïve (blue) donors 2 weeks postradiation. IL-1 $\beta$ concentrations were measured using ELISA. Values are expressed as means \pm SEM. *indicates $p \leq 0.05$, one-tailed t-test. IL, interleukin.

such effect was observed in GF irradiated mice following inoculation with radiation-induced microbiota as compared with inoculation with control microbiota (see online supplementary figure S1). This result supports a specific association between postradiation microbiota and induction of IL-1 $\beta$ secretion, rather than a nonspecific inflammatory response.

PCoA following sequencing of faecal samples from both groups 2 weeks postirradiation showed a significant difference 
in microbiota composition $(\mathrm{p}<0.05$, PCo1 scores comparison in weighted UniFrac index, Mann-Whitney U test; figure 6C).

LEfSe was used to identify differential OTU abundance between both GF mouse groups following bacterial inoculation and radiation and for comparison to microbiota of conventionally raised SPF irradiated mice (figure 6D). Phylum-level analysis demonstrated that GF mice inoculated with radiation-induced microbiota presented increased abundance of Bacteroidetes and Proteobacteria and decreased presence of Firmicutes (LDA score $>3.5, \mathrm{p}<0.01)$, similarly to the results obtained in the original model. Genus-level analysis revealed that Suterella and Parabacteroides were over-represented and members belonging to the Clostridiales order were decreased (LDA score $>3$, $\mathrm{p}<0.05$ ), similar to the original model. These results suggest similarity in microbial changes induced by radiation in both mouse groups.

\section{Radiation-induced dysbiosis transmits susceptibility to colitis}

To test whether the effect of postradiation microbiota was specific for radiation-induced damage, GF mice were inoculated with postradiation microbiota or control microbiota and treated with DSS for 6 days. As shown, GF mice inoculated with postradiation microbiota lost more weight (figure 7A), had more severe disease activity (figure $7 \mathrm{~B}$ ) and more damage as shown by histological analysis (figure $7 C)(p \leq 0.05$, one-tailed t-test). A trend for increased IL-1 $\beta$ levels was detected in this model as well ( $p=0.07$, one-tailed t-test, figure $7 D$ ). Similar to the radiation challenge, $\mathrm{TNF} \alpha$ expression was not induced by postirradiation microbiota compared with controls, reiterating the association of postradiation microbiota with IL-1 $\beta$ secretion. Taken together, these results demonstrate that radiation treatment affected faecal microbiota in a way that transmitted a proinflammatory signal, at least in response to radiation and DSS administration.

\section{IL-1 is a major mediator of colonic tissue damage in radiation-induced mucosal injury}

As noted, previous studies demonstrated that IL-1 $\beta$ expression had the strongest correlation with both RIS and the inflammatory score ${ }^{5}$ (see online supplementary table S2). The association with IL-1 $\beta$ secretion was further supported by the experiments conducted herein as well. However, such correlation may represent either a causative role for IL- $1 \beta$ in tissue damage or a protective compensatory response. Therefore, to further investigate this question, we tested whether IL-1 inhibition would protect or worsen the effects of radiation-induced damage. To this end, mice were treated with the IL-1 receptor antagonist, anakinra ${ }^{26}$
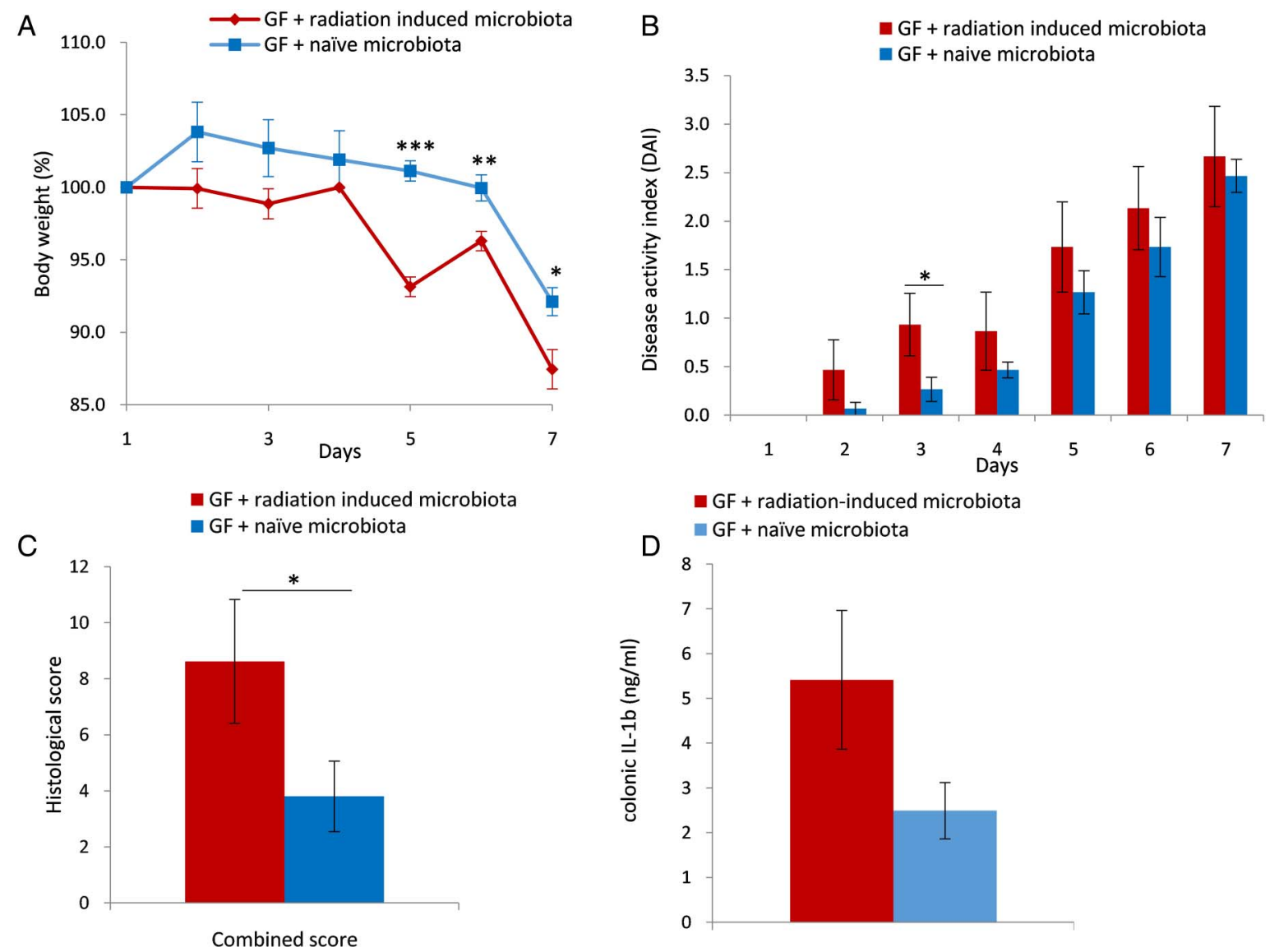

Figure 7 Radiation-induced microbiota exacerbates colonic dextran sulfate sodium (DSS)-induced injury in germ-free (GF) mice. Radiation-induced damage was evaluated based on (A) body weight and (B) the semiquantitative Disease Activity Index (DAI) 6 days after DSS $2.5 \%$ treatment in GF mice inoculated with microbiota from irradiated (red) and naïve (blue) donors. (C) Histological assessment of GF mice inoculated with faecal microbiota from irradiated (red) and naïve mice (blue) by the semiquantitative inflammatory combined histological score. The inflammatory score was evaluated 6 days post-DSS treatment and is represented as mean $\pm S E M$ (GF+radiation-induced microbiota: $n=5 ; G F+n a i ̈ v e ~ g r o u p: ~ n=5)$. ${ }^{*} \mathrm{p}<0.05 ;{ }^{* *} \mathrm{p}<0.01 ;{ }^{* *} \mathrm{p}<0.001$, one-tailed t-test. (D) Colonic IL-1 $\beta$ protein level in GF mice inoculated with microbiota from irradiated (red) and naïve (blue) donors, 6 days post-DSS treatment. IL-1 $\beta$ concentrations were measured using ELISA. Values are expressed as means \pm SEM. *indicates $\mathrm{p} \leq 0.05$, one-tailed t-test. IL, interleukin. 
and exposed to radiation. Treatment effects were assessed by histology and colonic cytokine expression (figure 8). As shown, anakinra treatment resulted in a lower RIS $(\mathrm{p} \leq 0.05$, Mann-Whitney U test, figure 8A), which was accompanied by reduced TNF $\alpha$ expression compared with irradiated control mice $(p<0.05$, Mann-Whitney $U$ test, figure $8 B)$. Taken together with the GF experiments, these observations support a sequence of events in which radiation alters the intestinal microbiota leading to increased host IL-1 $\beta$ secretion, which in turn aggravates radiation-induced tissue damage.

\section{DISCUSSION}

This study demonstrated that radiation treatment induced localised dysbiosis, which was associated with postradiation tissue damage as evident by the fact that the microbiome of radiated mice had pathogenic properties both in vitro and in vivo. We also show for the first time that dysbiosis induced by local radiation rendered GF recipient mice more susceptible to radiation damage and DSS-induced colitis. Dysbiosis played a role in intestinal inflammation and tissue damage, at least in part through IL-1 $\beta$ induction. Amelioration of tissue damage after blocking IL-1 reinforced IL-1 as a major driver of radiation-induced tissue damage.

Similar to a previous study, ${ }^{5}$ inflammatory cytokine expression correlated with the RIS. We observed different cytokine clusters and tissue damage corresponding to the 2 and 6-week time points. These changes were not isolated, since changes in the microbial composition, paralleled with the RIS and cytokine profile in the two time points, were observed. Comparison of faecal microbial compositions between irradiated and control mice at the two time points revealed that the microbiota significantly differed following local radiation treatment. These results corroborate findings of others, who showed that radiotherapy is associated with microbial compositional changes, using different methodologies including bacterial cultures, fingerprinting, clone libraries and recently pyrosequencing. ${ }^{14-17}$

A unique microbial signature was observed at the tested time points. By analysing clustering patterns, it was apparent that microbial changes appeared gradually, with the 2 -week postradiation time point being most similar to the bacterial composition before radiation treatment. The predominant change in bacterial composition occurred at the chronic phase 6 weeks postradiation. The findings are consistent with those reported by Nam et $a l^{17}$ who also observed a gradual response of gut microbiota postradiation in patients with gynaecological cancer, reaching a maximum 1-3 months postradiation.

The associations between the unique microbiota patterns and inflammatory parameters during different postradiation disease stages raised the question of cause and effect. There are contradicting data regarding whether and how the microbiota affects radiation-induced tissue damage. Using a total body radiation model, it was shown that administration of Salmonella flagellin had a protective effect from GI radiation damage by suppressing apoptosis. ${ }^{27}$ However, a study using GF mice demonstrated that these mice were markedly resistant to lethal radiation. ${ }^{28}$ Additionally, several studies in both rodents and humans have shown that administration of specific bacterial species, mainly Lactobacillus, administered as a probiotic reduced postradiation intestinal damage. ${ }^{29}{ }^{30}$ However, other studies suggested the lack of a significant protective effect. ${ }^{31} 32$

In vitro experiments in the current study demonstrated that the dysbiotic microbiota exerted a direct proinflammatory effect on the epithelial cells, as shown by increased expression of TNF $\alpha$ and IL-1 $\beta$ following co-culture compared with effects exerted by naïve microbiota. Previous studies demonstrated that epithelial cells play a central role in bacterial sensing by expressing receptors for microbial-associated patterns, which can trigger expression of an array of chemokines, cytokines and effectors of innate immunity. ${ }^{33} 34$ Due to the importance of the epithelial barrier in the inflammatory response, the results

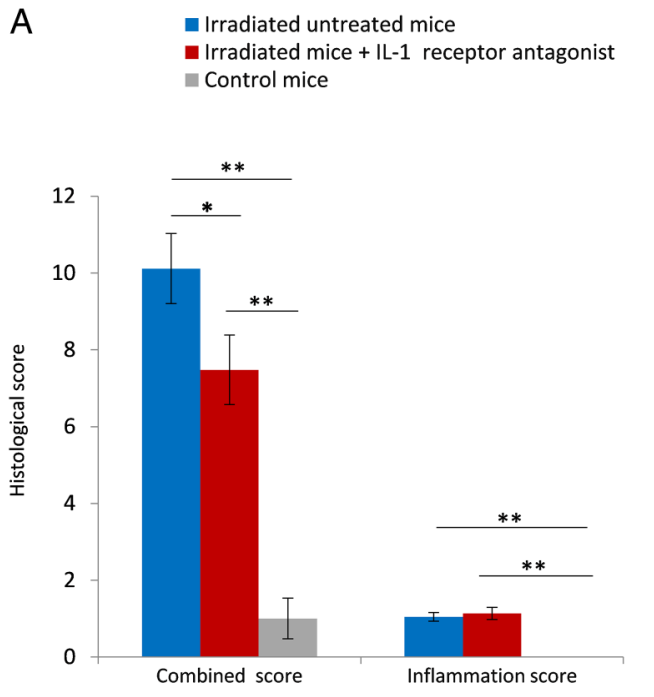

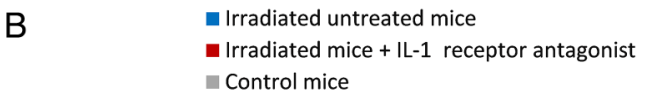

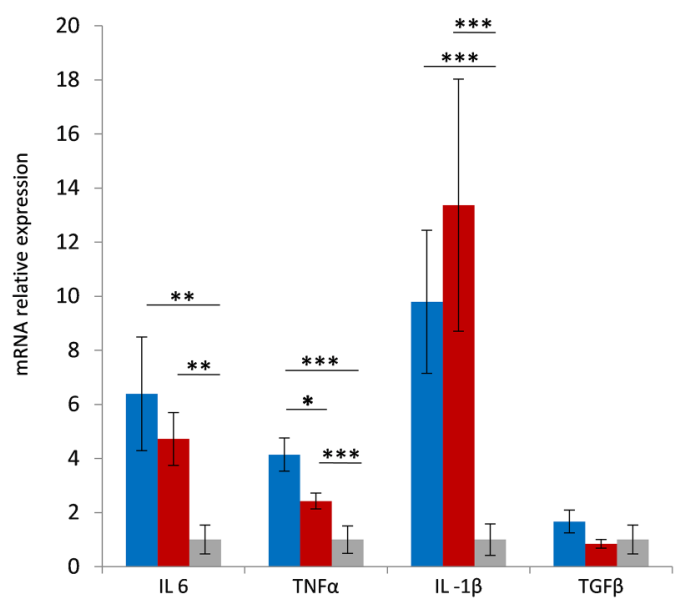

Figure 8 IL-1 blockage reduces postradiation colonic damage and cytokine expression. Mice were treated with anakinra following radiation treatment. At 6 weeks postradiation, mice were evaluated by histological assessment (A) and local colonic cytokine expression (B). Results from irradiated anakinra treated mice are shown in red, from irradiated untreated mice in blue and from naïve non-irradiated mice in grey. Expression of colonic cytokines was assessed by quantitative PCR of colon samples. Data were normalised to the non-irradiated mouse group. Histopathology was analysed to determine the semiquantitative radiation injury score. Data are represented as mean $\pm S E M$ (anakinra treated group: $n=22$, untreated group: $n=22$; naïve non-irradiated group: $n=4$ ). Data shown were pooled from two independent experiments. ${ }^{*} p<0.05 ;{ }^{* *} p<0.01$; ${ }^{* * *} p<0.001$, Mann-Whitney U test. IL, interleukin. 
suggest a causative role for the microbial dysbiosis in the chronic postradiation phase. The complementary set of experiments using GF mice indicated that postradiation microbiota predisposed the mice to both radiation damage and an inflammatory trigger (DSS), and that this phenomenon was accompanied by induction of intestinal IL-1 $\beta$ secretion. Taken together with the in vitro observations, the data suggest a sequence of events in which radiation induces dysbiosis, which in turn triggers mucosal cytokine release, particularly IL-1 $\beta$, aggravating mucosal damage; this is supported by the amelioration of tissue damage following IL-1 receptor antagonist administration, thereby identifying the change in microbiota as the primary event in this model. The fact that these effects occurred in several mouse strains further strengthens this conclusion. These data are in agreement with other studies in which bacteria were shown to transmit an environmental signal affecting an ongoing biological process. For example, Blanton $e t$ al $l^{35}$ demonstrated that microbiota from Malawian undernourished children transmitted an impaired growth phenotype to GF mice fed Malawian diet.

Analysis of the radiation-induced microbial changes revealed several bacterial genera that were over-represented in the injured colon and were common to both conventionally raised irradiated mice and irradiated GF mice inoculated with radiation-induced microbiota, which showed more severe disease manifestation. The most significant and consistent in both original and GF mouse models is the expansion of Parabacteroides and Sutterella spp., the latter was also attributed to significant increased abundance of the Proteobacteria phylum. Sutterella was reported to induce an IgA low phenotype by degradation of the secretory component of SIgA as well as IgA itself, which in turn increased susceptibility to DSS-induced colitis. This effect was transmissible by faecal transplantation. ${ }^{36}$ The abundance of Sutterella in the radiation model herein suggests that radiation may drive inflammation through similar mechanisms, hence promoting the inflammatory response and aggravating tissue damage.

The use of anakinra does not allow differentiation between IL- $1 \alpha$ and IL-1 $\beta$ effects. However, several recent studies support our findings which attribute an important role for IL-1 $1 \beta$ in the context of radiation. One study demonstrated that IL- $1 \beta$ exacerbated mucositis in a mouse model through tight junction disruption. ${ }^{37}$ Another study reported that both IL- $1 \alpha$ and IL- $1 \beta$ play a role in radiodermatitis, but IL-1 $\beta$ had greater impact on the inflammatory process. ${ }^{38}$ Of note, although $\mathrm{TNF} \alpha$ was also induced in the in vitro model, only IL-1 $\beta$ was induced by the dysbiotic microbiota in GF mice compared with controls, further pointing to their unique association. Taken together, the findings support IL-1 $\beta$ as a key molecule in the context of radiation-induced intestinal damage. Notably, anakinra is FDA approved for human use and the present findings may support exploration of its potential therapeutic effect in the context of radiation.

In conclusion, the results of this study demonstrate radiation-induced changes in the microbiome and their potential impact on inflammation. We also demonstrated a transmissible propensity for inflammation and radiation damage imparted by the dysbiotic microbiota. Our results show for the first time that the altered microbiota induced mucosal IL- $1 \beta$ secretion, which contributed at least in part to radiation-induced colonic damage. Collectively, the findings suggest that microbiota manipulation or direct inhibition of IL-1 may constitute a potential therapeutic approach for reducing intestinal radiation-induced damage.

\section{Author affiliations}

1Department of Gastroenterology, Rambam Health Care Campus, Ruth and Bruce Rappaport School of Medicine, Technion-Israel Institute of Technology, Haifa, Israel ${ }^{2}$ Faculty of Biotechnology and Food Engineering, Technion-Israel Institute of Technology, Haifa, Israel

${ }^{3}$ Department of Pathology, Rambam Medical Center, Haifa, Israel

${ }^{4}$ Radiation Unit, Department of Oncology, Rambam Medical Center, Haifa, Israel

${ }^{5}$ Faculty of Medicine, Bar-Ilan University, Safed, Israel

${ }^{6}$ Division of Gastroenterology, University of Miami, Miller School of Medicine, Miami, USA

Contributors $Y C, Y K$ and SG-V: study concept and design; $S G-V, A B, K G, A D, R D$ and OZ: acquisition of data; YC, YK, SG-V, AB, YD-P and RD: analysis/interpretation of data; ES: histological analysis; AN and SD: irradiation procedures; YC, YK, MTA, OK and OZ: critical revision of the manuscript for important intellectual content; YC, YK and SG-V: drafting of the manuscript; $Y C$ and YK: study supervision.

Funding This work was supported by grants from the Israel Cancer Association (grant 2017562) and BSF USA-Israel Binational Science Foundation (grant 2009076).

\section{Competing interests None declared.}

Provenance and peer review Not commissioned; externally peer reviewed.

\section{REFERENCES}

1 Churnratanakul S, Wirzba B, Lam T, et al. Radiation and the small intestine. Future perspectives for preventive therapy. Dig Dis 1990;8:45-60.

2 Keefe DMK. Gastrointestinal mucositis: a new biological model. Support Care Cancer 2004;12:6-9.

3 Jung $E$, Perrone EE, Brahmamdan $P$, et al. Inhibition of Intestinal Epithelial Apoptosis Improves Survival in a Murine Model of Radiation Combined Injury. PLOS ONE 2013;8:e77203.

4 Indaram AVK, Visvalingam V, Locke $M$, et al. Mucosal cytokine production in radiation-induced proctosigmoiditis compared with inflammatory bowel disease. Am J Gastroenterol 2000;95:1221-5.

5 Symon Z, Goldshmidt Y, Picard O, et al. A murine model for the study of molecular pathogenesis of radiation proctitis. Int J Radiat Oncol Biol Phys 2010;76:242-50.

6 Citrin $\mathrm{D}$, Cotrim $\mathrm{AP}$, Hyodo $\mathrm{F}$, et al. Radioprotectors and mitigators of radiation-induced normal tissue injury. Oncologist 2010;15:360-71.

7 Begg AC, Stewart FA, Vens C. Strategies to improve radiotherapy with targeted drugs. Nat Rev Cancer 2011;11:239-53.

8 Baskar R, Lee KA, Yeo R, et al. Cancer and radiation therapy: current advances and future directions. Int J Med Sci 2012;9:193-9.

9 Shadad AK, Sullivan FJ, Martin JD, et al. Gastrointestinal radiation injury: symptoms, risk factors and mechanisms. World J Gastroenterol 2013;19:185-98.

10 Barcellos-Hoff MH, Park C, Wright EG. Radiation and the microenvironmenttumorigenesis and therapy. Nat Rev Cancer 2005;5:867-75.

11 Tsirigotis P, Triantafyllou K, Girkas K, et al. Keratinocyte growth factor is effective in the prevention of intestinal mucositis in patients with hematological malignancies treated with high-dose chemotherapy and autologous hematopoietic SCT: a video-capsule endoscopy study. Bone Marrow Transplant 2008:42:337-43.

12 Johnson $L B$, Riaz AA, Adawi $D$, et al. Radiation enteropathy and leucocyte-endothelial cell reactions in a refined small bowel model. BMC Surg 2004;4:10.

13 Heimesaat MM, Nogai A, Bereswill S, et al. MyD88/TLR9 mediated immunopathology and gut microbiota dynamics in a novel murine model of intestinal graft-versus-host disease. Gut 2010;59:1079-87.

14 Jenq RR, Ubeda C, Taur Y, et al. Regulation of intestinal inflammation by microbiota following allogeneic bone marrow transplantation. J Exp Med 2012:209:903-11.

15 Manichanh C, Varela E, Martinez C, et al. The gut microbiota predispose to the pathophysiology of acute postradiotherapy diarrhea. Am J Gastroenterol 2008; 103:1754-61

16 Husebye $\mathrm{E}$, Skar $\mathrm{V}$, Hoverstad $\mathrm{T}$, et al. Abnormal intestinal motor patterns explain enteric colonization with gram-negative bacilli in late radiation enteropathy. Gastroenterology 1995;109:1078-89.

$17 \mathrm{Nam}$ YD, Kim HJ, Seo JG, et al. Impact of pelvic radiotherapy on gut microbiota of gynecological cancer patients revealed by massive pyrosequencing. PLOS ONE 2013;8:e82659.

18 Denham JW, Hauer-Jensen M, Kron T, et al. Treatment-time-dependence models of early and delayed radiation injury in rat small intestine. Int J Radiat Oncol Biol Phys 2000;48:871-87

19 Demirer S, Aydintug S, Aslim B, et al. Effects of probiotics on radiation-induced intestinal injury in rats. Nutrition 2006;22:179-86.

20 Akgun E. Caliskan C, Celik HA, et al. Effects of $\mathrm{N}$-acetylcysteine treatment on oxidative stress in acetic acid-induced experimental colitis in rats. J Int Med Res 2005;33:196-206. 
21 Caporaso JG, Kuczynski J, Stombaugh J, et al. QIIME allows analysis of high-throughput community sequencing data. Nat Methods 2010;7:335-6.

22 Eckmann L, Jung HC, Schürer-Maly C, et al. Differential cytokine expression by human intestinal epithelial cell lines: regulated expression of interleukin 8. Gastroenterology 1993;105:1689-97.

23 Murthy SN, Cooper HS, Shim H, et al. Treatment of dextran sulfate sodium-induced murine colitis by intracolonic cyclosporin. Dig Dis Sci 1993;38:1722-34.

24 Lozupone C, Knight R. UniFrac: a new phylogenetic method for comparing microbial communities. Appl Environ Microbiol 2005;71:8228-35.

25 Segata N, Izard J, Waldron L, et al. Metagenomic biomarker discovery and explanation. Genome Biol 2011;12:R60.

26 Maeda S, Hsu LC, Liu H, et al. Nod2 mutation in Crohn's disease potentiates NF-kappaB activity and IL-1beta processing. Science 2005;307:734-8.

27 Burdelya LG, Krivokrysenko VI, Tallant TC, et al. An agonist of toll-like receptor 5 has radioprotective activity in mouse and primate models. Science 2008;320:226-30.

28 Crawford PA, Gordon Jl. Microbial regulation of intestinal radiosensitivity. Proc Natl Acad Sci USA 2005;102:13254-9

29 Delia G, Donato V, Frosina P, et al. .. Use of probiotics for prevention of radiation-induced diarrhea. World I Gastroenterol 2007:13:912-5.

30 Chitapanarux I, Chitapanarux T, Traisathit P, et al. Randomized controlled trial of live lactobacillus acidophilus plus bifidobacterium bifidum in prophylaxis of diarrhea during radiotherapy in cervical cancer patients. Radiat Oncol 2010;5:31.
31 Urbancsek H, Kazar T, Mezes I, et al. Results of a double-blind, randomized study to evaluate the efficacy and safety of Antibiophilus in patients with radiation-induced diarrhoea. Eur J Gastroenterol Hepatol 2001;13:391-6.

32 Giralt J, Regadera JP, Verges $\mathrm{R}$, et al. Effects of probiotic Lactobacillus casei DN-114 001 in prevention of radiation-induced diarrhea: results from multicenter, randomized, placebo-controlled nutritional trial. Int I Radiat Oncol Biol Phys 2008;71:1213-19.

33 Artis D. Epithelial-cell recognition of commensal bacteria and maintenance of immune homeostasis in the gut. Nat Rev Immunol 2008;8:411-20.

34 Wells JM, Rossi O, Meijerink M, et al. Epithelial crosstalk at the microbiota-mucosal interface. Proc Natl Acad Sci USA2011;108(Suppl 1):4607-14.

35 Blanton LV, Charbonneau MR, Salih T, et al. Gut bacteria that prevent growth impairments transmitted by microbiota from malnourished children. Science 2016;351.

36 Moon C, Baldridge MT, Wallace MA, et al. Vertically transmitted faecal IgA levels determine extra-chromosomal phenotypic variation. Nature 2015; 521:90-3.

37 Kanarek N. Grivennikov SI, Leshets M, et al. Critical role for IL-1beta in DNA damage-induced mucositis. Proc Natl Acad Sci USA 2014;111:E702-11.

38 Janko $\mathrm{M}$, Ontiveros F, Fitzgerald TJ, et al. IL-1 generated subsequent to radiation-induced tissue injury contributes to the pathogenesis of radiodermatitis. Radiat Res 2012;178:166-72. 